\title{
Extracting features buried within high density Atom Probe Point Cloud data through Simplicial Homology
}

\author{
Srikant Srinivasan, Kaustubh Kaluskar, Scott Broderick and Krishna Rajan. \\ Institute of Combinatorial Discovery, Department of Materials Science and Engineering, Iowa State \\ University, Ames, IA 50011-2300
}

\begin{abstract}
Feature extraction from Atom Probe Tomography (APT) data is usually performed by repeatedly delineating iso-concentration surfaces of a chemical component of the sample material at different values of concentration threshold, until the user visually determines a satisfactory result in line with prior knowledge. However, this approach allows for important features, buried within the sample, to be visually obscured by the high density and volume $\left(\sim 10^{7}\right.$ atoms $)$ of APT data. This work provides a data driven methodology to objectively determine the appropriate concentration threshold for classifying different phases, such as precipitates, by mapping the topology of the APT dataset using a concept from algebraic topology termed persistent simplicial homology. A case study of Sc precipitates in an Al-Mg-Sc alloy is presented demonstrating the power of this technique to capture features, such as precise demarcation of Sc clusters and $\mathrm{Al}$ segregation at the cluster boundaries, not easily available by routine visual adjustment.
\end{abstract}

\section{Introduction}

Atom Probe Tomography (APT) presents [1-5] enormous potential in probing the sub-nanometer character of materials. However, recovering this information from data generated at such high spatial resolution naturally presents the concomitant challenge of interpreting very high volume and high density data $[6,7]$. Even small data collections involve $\sim 10^{7}$ atoms and the standard procedure of visualizing this data set to isolate features can allow important ones, such as precipitates, to be easily lost within the high volume of data. Feature extraction typically requires drawing iso-concentration surfaces [8, 9] at a particular concentration threshold, then visually exploring the data space to probe for various features, and repeating the procedure over an entire range of concentration threshold values. Following up on our earlier work in rendering such high volume APT data to aid in feature extraction [10-12] we now provide an alternate data driven approach of objectively classifying different phases such as precipitates by mapping the topology of the APT dataset using concepts from algebraic topology, namely, simplicial homology [13-15].

Topology is inherently a classification system that deals with qualitative geometric information. This includes the study of what the connected components of a space are and their connectivity information in 
different dimensions of space [16]. Metric properties such as the position of a point, the distance between points, or the curvature of a surface, are irrelevant to topology. Thus, a circle and a square have the same topology although they are geometrically different. Such topological invariants can be represented by simplicial complexes, which are combinatorial objects that can represent spaces and separate the topology of a space from its geometry [14]. Examples of simplices include a point (0-dimensional simplex), a line segment (1-dimensional simplex), a triangle (2-dimensional simplex) and a tetrahedron (3-dimensional simplex).

Simplicial homology is a process that provides information about the simplicial complex by the number of cycles (a type of hole) it contains. One of its informational outcomes are Betti numbers which record the topological invariants of an object, invariants such as the number of connected components, holes, tunnels, or cavities [17]. While a structure can have infinite shapes, many of which cannot be quantified, it can have only limited topological features depending on its dimension. For example, in three dimensions (3D), a structure can be simply connected, or it can be connected such that a tunnel passes through it, or it can be connected to itself such that it encloses a cavity, or it can remain unconnected. Thus, we can characterize the topology of a structure by counting the number of simply connected components, number of tunnels and number of cavities denoted by Betti numbers $\beta_{0}, \beta_{1}$ and $\beta_{2}$.

\section{Methods}

When dealing with point cloud data representing physical structures, such as the APT data, the number and type of topological invariants clearly depends on the degree of connectivity between the various points, established through some metric such as distance. The determination of which points to connect can be addressed by defining a sphere of radius ' $\varepsilon$ ' around each point and connecting it to all those other points that lie within this sphere. Again there could be a measure of arbitrariness in determining the appropriate value of $\varepsilon$. A small change in $\varepsilon$ for randomly distributed points can quickly change the underlying topology due to statistical noise, thus changing the Betti numbers of the structure. The challenge is to determine the appropriate value of $\varepsilon$ that corresponds to a meaningful feature. A powerful technique to overcome this problem is persistent homology [18], so termed because it is based on the idea that betti numbers relating to random distribution of data points and noise cannot persist as we vary $\varepsilon$. The value of $\varepsilon$ is gradually increased from 0 and the numbers of different topological components that appear and disappear are tracked for changing $\varepsilon$. This process is called filtration. Only those topological invariants that represent true features in the underlying data will remain unaffected by small changes in $\varepsilon$.

An example of the evolution of Betti numbers with changing $\varepsilon$ is shown with 2-D data in Fig. 1. The data points are obtained by sampling 5 points each, at regular intervals, from 3 parallel lines. At $\varepsilon=0$, there are 
15 vertices or 0 -simplices forming 15 independent components with $\beta_{0}=15$. As the distance threshold for connectivity to adjacent points is increased the vertices connect to their first nearest neighbors resulting in 3 connected components, representing the 3 parallel lines or $\beta_{0}=3$. As $\varepsilon$ is increased further, each point now connects to its second nearest neighbor thus forming 8 squares, each square representing a hole. The whole structure is a single connected component, thus $\beta_{0}=1$ and $\beta_{l}=8$. Finally for further increase in $\varepsilon$ each vertex now connects to its third nearest neighbor forming triangles, thus, closing the 8 holes. Therefore, $\beta_{0}=1$ and $\beta_{l}=0$.

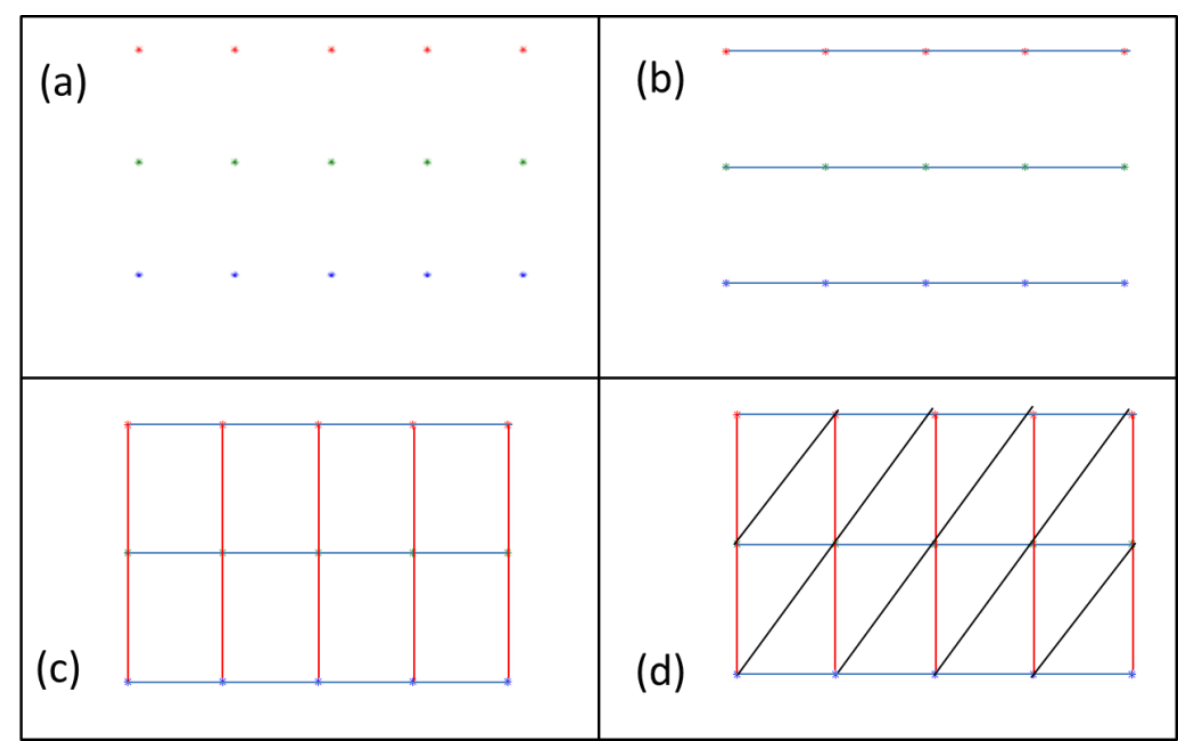

Fig. 1: Persistence in a 2-D point set sampled from a rectangular grid. (a) 15 vertices or 0 -simplices forming 15 independent components with $\beta_{0}=15$. (b) As the distance threshold is increased the vertices connect to their first nearest neighbors resulting in 3 connected components ( 3 parallel lines) or $\beta_{0}=3$. (c) Each point now connects to its second nearest neighbor thus forming 8 squares, each square representing a hole. The whole structure is a single connected component, thus $\beta_{0}=1$ and $\beta_{I}=8$. (d) Each vertex is now connected to its third nearest neighbor forming triangles, thus, closing the 8 holes. Therefore, $\beta_{0}=1$ and $\beta_{1}=0$.

The persistence of different topological features can be recorded as barcodes [Fig. 2] grouped according to each Betti number. The horizontal axis represents the parameter $\varepsilon$ or the range of connectivity around points in the point cloud, while the vertical axis captures the number of topological components present in the point cloud at each interval of $\varepsilon$ for $\beta_{0}$ (Fig. 2(a)) and $\beta_{1}$ (Fig. 2(b)). Initially there are 15 vertices or independent components with $\beta_{0}=15$ at $\varepsilon=0$. As the distance threshold is increased the vertices connect to their first nearest neighbors forming 3 parallel lines at $\varepsilon=0.5$ resulting in the 15 bars on the barcode plot collapsing into 3 or $\beta_{0}=3$. If the 3 lines were sufficiently far apart, then the value of $\beta_{0}=3$ would have persisted for a long duration. However, since the separation between adjacent points is comparable to the separation between points on each line, at $\varepsilon=1$ the points on different lines are interconnected and the 
whole structure is a single connected component, thus, $\beta_{0}=1$ thereafter for $\varepsilon \rightarrow \infty$. It should be noted that eventually for $\varepsilon \rightarrow \infty$, the resultant structure will always have $\beta_{0}=1$ since every vertex will eventually connect to every other one. There has to be some knowledge of the appropriate range for $\varepsilon$, such as the interatomic distance when dealing with raw atom probe data, or voxel length if the data has been voxelized. For $\beta_{1}$, at $\varepsilon=1$ there are 8 squares, each square representing a hole. Thus, $\beta_{l}=8$. However, as soon as each vertex connected to its third nearest neighbor, forming triangles, the holes are closed again leading to $\beta_{l}=0$. The persistence of features is a measure of whether these features are actually present in the data or if they are artifacts appearing at certain intervals. The 8 holes on the $\beta_{1}$ appear for a very short duration and immediately close up.
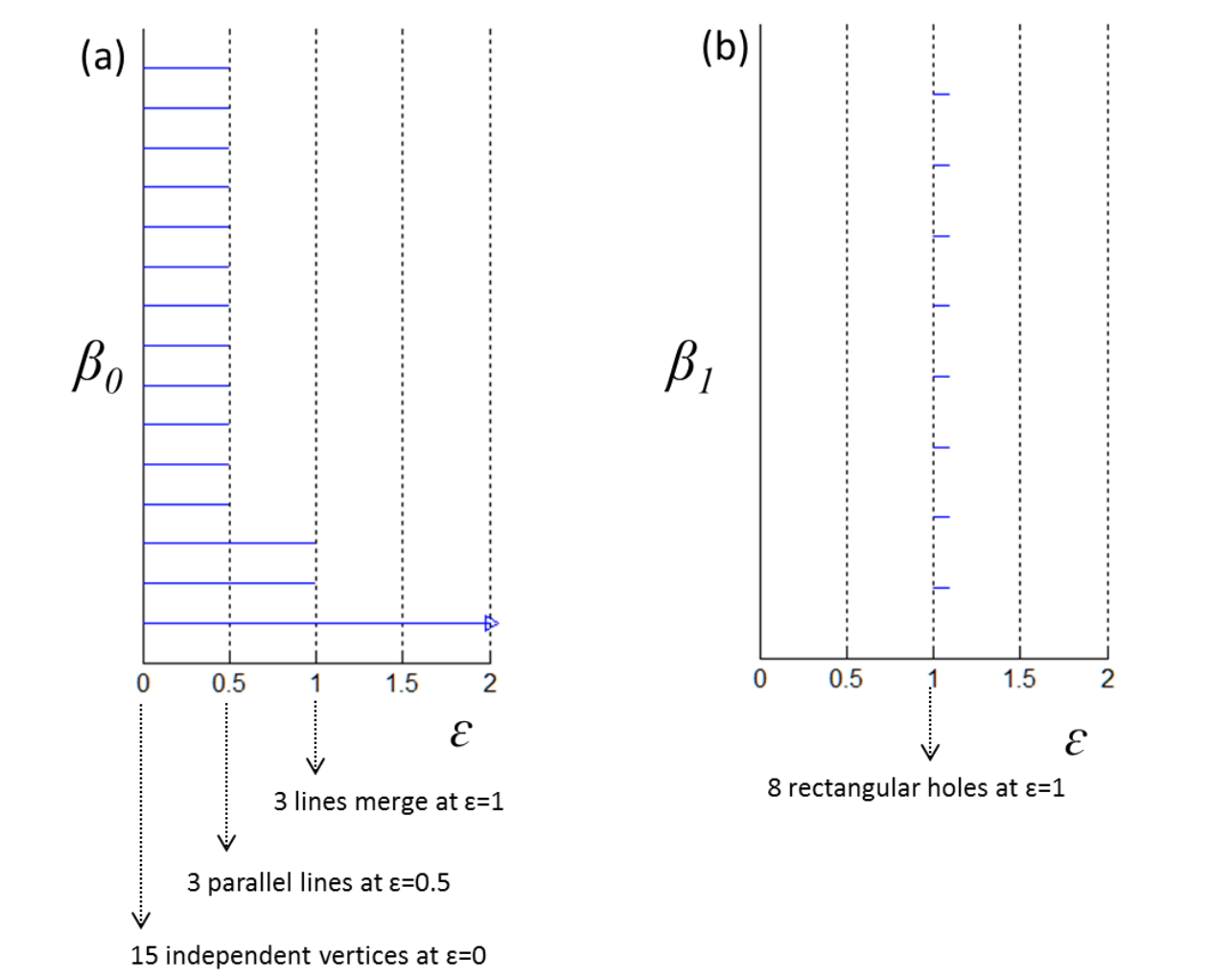

Fig. 2: Barcode representation of the persistent homology process in Fig. 1. (a) The figure on the left represents $\beta_{0}$. Initially there are 15 vertices or independent components with $\beta_{0}=15$ at $\varepsilon=0$. As the distance threshold is increased the vertices connect to their first nearest neighbors at $\varepsilon=0.5$ resulting in 15 bars collapsing into 3 or $\beta_{0}=3$ representing 3 parallel lines. At $\varepsilon=1$ the lines are interconnected and the whole structure is a single connected component, thus $\beta_{0}=1$ thereafter for $\varepsilon \rightarrow \infty$. (b) The figure on the right represents the barcode corresponding to $\beta_{1}$. At $\varepsilon=1$ there are 8 squares, each square representing a hole, thus, $\beta_{I}=8$. However as soon as each vertex connected to its third nearest neighbor, forming triangles, the holes are closed again leading to $\beta_{1}=0$.

For the 3D APT data, the sheer number of data points can potentially make the Betti number computation intractable. To overcome this we employ the witness complex[19] construction, which allows us to build a simplicial complex using landmark points sampled from a point cloud data set, carefully chosen to 
represent the original data. This is demonstrated in Fig. 3 for points sampled from a sphere. The original sampling [Fig. 3(a)] contains about 2000 points and by downsampling [Fig. 3(b)] we can choose about 200 points, which are appropriately spaced.

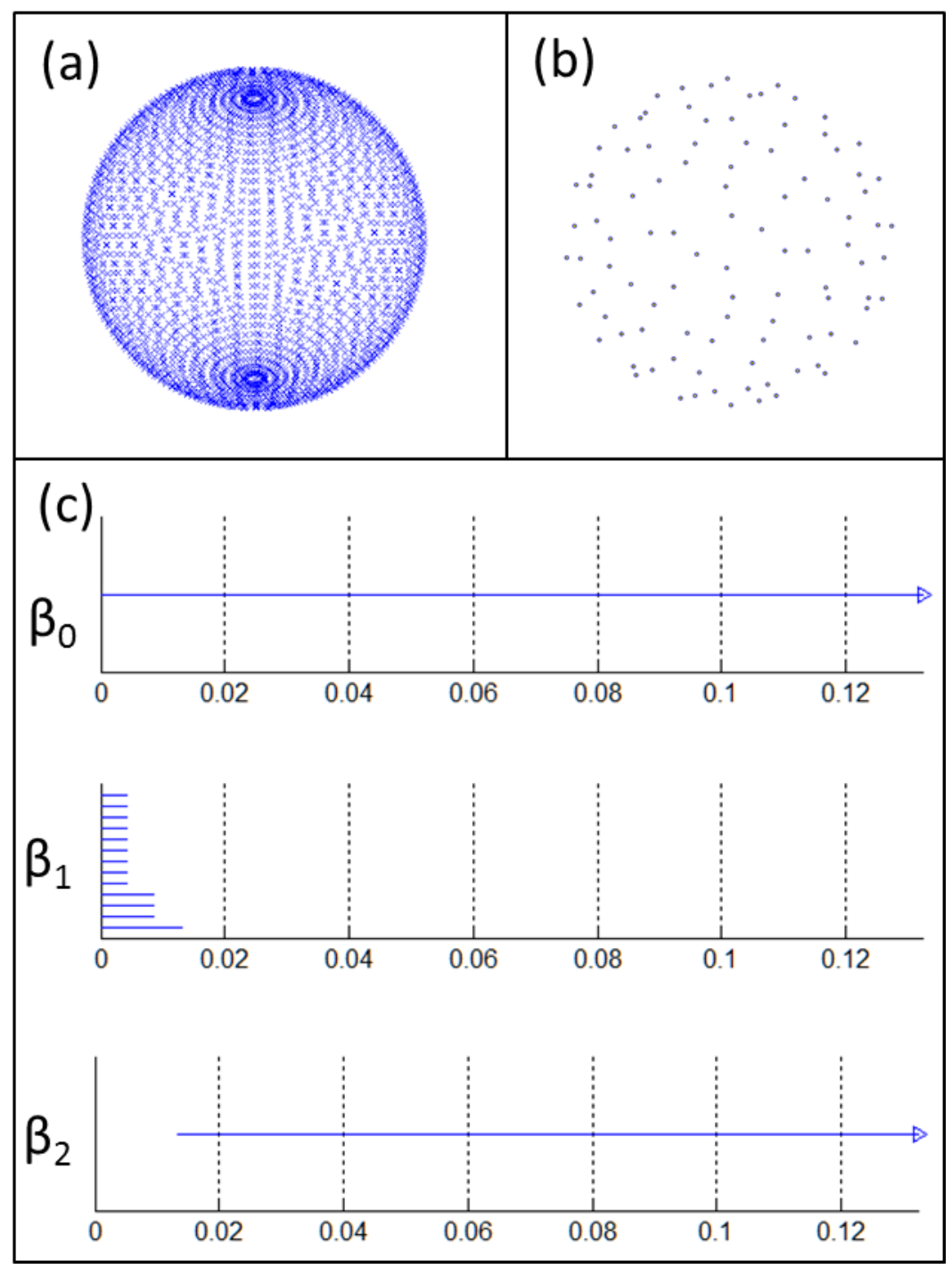

Fig. 3: Persistent homology using Witness complex construction of a sphere. (a) the original point cloud data consisting of 2000 points. (b) Extracting 1\% of the original data points as landmark points which are equally spaced from each other as much as possible (Note: equal spacing not readily visible due to projection onto a 2-D plane) (c) The barcode using the witness complex shows that initially as the points connect to their nearest neighbors, numerous meandering paths are created across the surface of the sphere. Due to equidistant spacing every point is connected to at least one other points so there is only one connected component. Many of the points form closed loops leading to multiple bars on the $\beta_{1}$ plot. However, as the connectivity is increased these loops fail to persist and the right answer of $\left(\beta_{0}, \beta_{1}, \beta_{2}\right)=(1$, $0,1)$ is obtained, representing 1 connected component and 1 cavity. 
The points are chosen to be random or evenly spaced. The advantage of even spacing is that it minimizes the probability of statistical noise while on the other hand it necessarily includes outliers. For selecting fine features in APT data such as precipitates we employ the evenly spaced selection of points since random sampling can introduce spurious nanoclusters, which may be misleading. Fig. 3(c) shows the filtration barcode using the witness complex, with evenly spaced points. The barcode shows that initially as the points connect to their nearest neighbors, numerous meandering paths are created across the surface of the sphere. Due to equidistant spacing every point is connected to at least one other points so there is only one connected component. However, many of the points form closed loops leading to multiple bars on the $\beta_{1}$ plot. In this case, due to a high density of points on the sphere and choice of step size of the filtration parameter, the starting value of filtration already exceeds the distance between nearest neighbors so the individual points do not show up on the $\beta_{0}$ plot. As the connectivity is increased these loops fail to persist and the right answer of $\left(\beta_{0}, \beta_{1}, \beta_{2}\right)=(1,0,1)$ is obtained, representing 1 connected component and 1 cavity

APT data: APT experiments were carried out on Al-95/ Mg-4.5/ Sc-0.4 alloy samples. The sample, containing $\sim 20$ million atoms, shows that the Sc atoms tend to cluster into precipitate regions with an average Sc concentration of $\sim 50 \%$ accompanied with Al depletion. Fig. 4 shows a blowup of a region containing precipitates of $\mathrm{Sc}$ inside the $\mathrm{Al}-\mathrm{Mg}$ matrix. For clarity two representations of the same region are shown, one with all chemistries and the other with the $\mathrm{Al}$ atoms removed from the image, showing that $\mathrm{Sc}$ is present in the precipitates and $\mathrm{Mg}$ is in the matrix. 


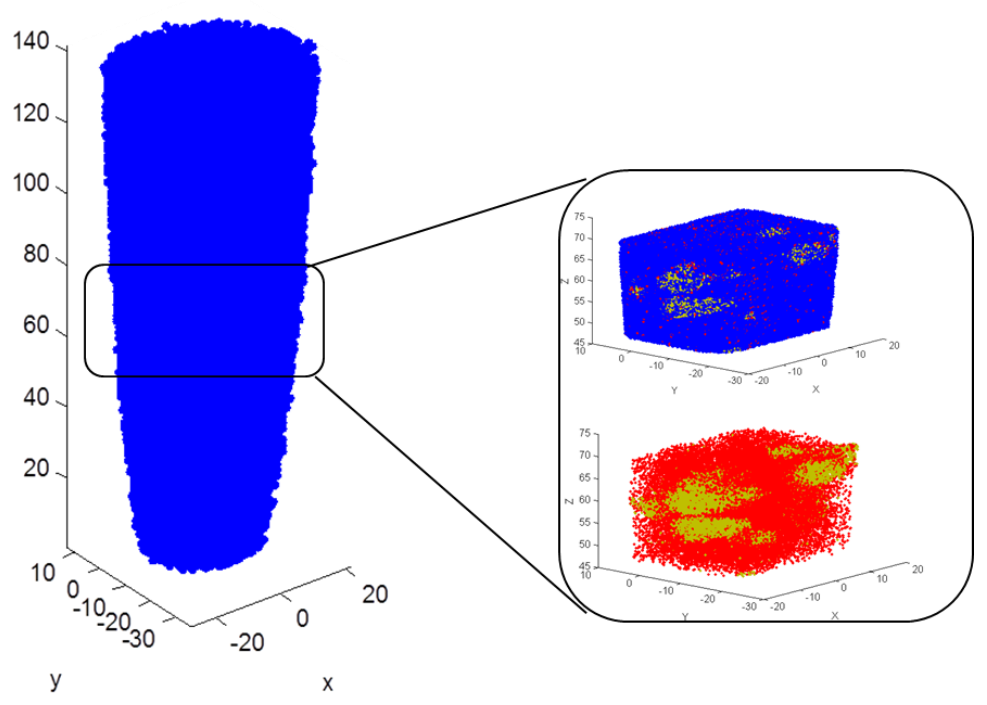

Fig. 4: APT point cloud data from Al-Mg-Sc sample. For clarity the regions with Sc precipitates are blown up in the inset. The blue, red and green dots represent $\mathrm{Al}, \mathrm{Mg}$ and $\mathrm{Sc}$ respectively. Two representations of the same region are shown, one with all chemistries and the other with the $\mathrm{Al}$ atoms removed from the image, showing that $\mathrm{Sc}$ is present in the precipitates and $\mathrm{Mg}$ is in the matrix.

\section{Results and Analysis}

For the purpose of computing the topological features of different chemistries from the reconstructed APT data on Al-Mg-Sc, it is divided in 3-D boxes called voxels [20, 21]. Voxelization allows the demarcation of boundaries for computation of local density of different chemical constituents of the sample. Using the density information of the voxels allows the representation of data in the form of isoconcentration surfaces. The edge length of each voxel was set at $1.5 \mathrm{~nm}$ resulting in about 6000 voxels. The atomic point cloud data is, thus, downsampled from 20 million to 6000, by using the coordinates of center of the voxels to represent the original data.

The filtration of voxelized data based on the density information is carried out using the tool Javaplex [22]. Unlike the typical filtration process where $\varepsilon$, the distance metric of connectivity, is varied for a fixed number of data points, the approach we follow here is to set $\varepsilon$ to a fixed value equal to the spacing between the centers of two adjacent voxels. This is because the process of voxelization bins the data into a uniform volumetric grid, circumventing the application of varying $\varepsilon$. Instead filtration is carried out with respect to concentration. Each voxel is associated with a certain value of local concentration and by varying the value of concentration as our filtration parameter, our underlying dataset provides a different 
set of voxels corresponding to each concentration value chosen. We vary the concentration threshold of each element (Al, Mg, Sc) independently in Figs. [5-7] to show how the process evolves.

Fig. 5 shows the evolution of Betti numbers for varying Sc concentration ' $\delta$ ' over the range $\delta \in[0,0.8]$. At each value of Sc concentration threshold ' $\delta$ ', the set of voxels that have a Sc concentration of $\delta \pm 0.02$ are chosen from the original data set (inset). The topology calculation is performed on this set of voxels. As mentioned above the parameter $\varepsilon$ takes on a fixed value, which we set $\varepsilon=$ voxel edge length implying that we are computing for nearest neighbor connectivity. At each step of the simulation over the range of $\delta$, all voxels that have a Sc concentration of $\delta \pm 0.02$ are extracted from the data set. Among these, those voxels that form nearest neighbors within a distance of the voxel edge length are connected together. Having established the connections within this set of voxels, the analysis of topology is performed, namely, how many of these voxels form singly connected components (e.g. groups of voxels that form clusters) or how many form cavities (representing regions forming the boundaries of clusters). The addition of \pm 0.02 to $\delta$ is a computational construct to account for statistical noise in the distribution of atoms. Without the inclusion of this small range, statistical noise can artificially disconnect neighboring voxels due to an infinitesimal difference in concentration distribution and introduce artifacts in the number of topological components. 


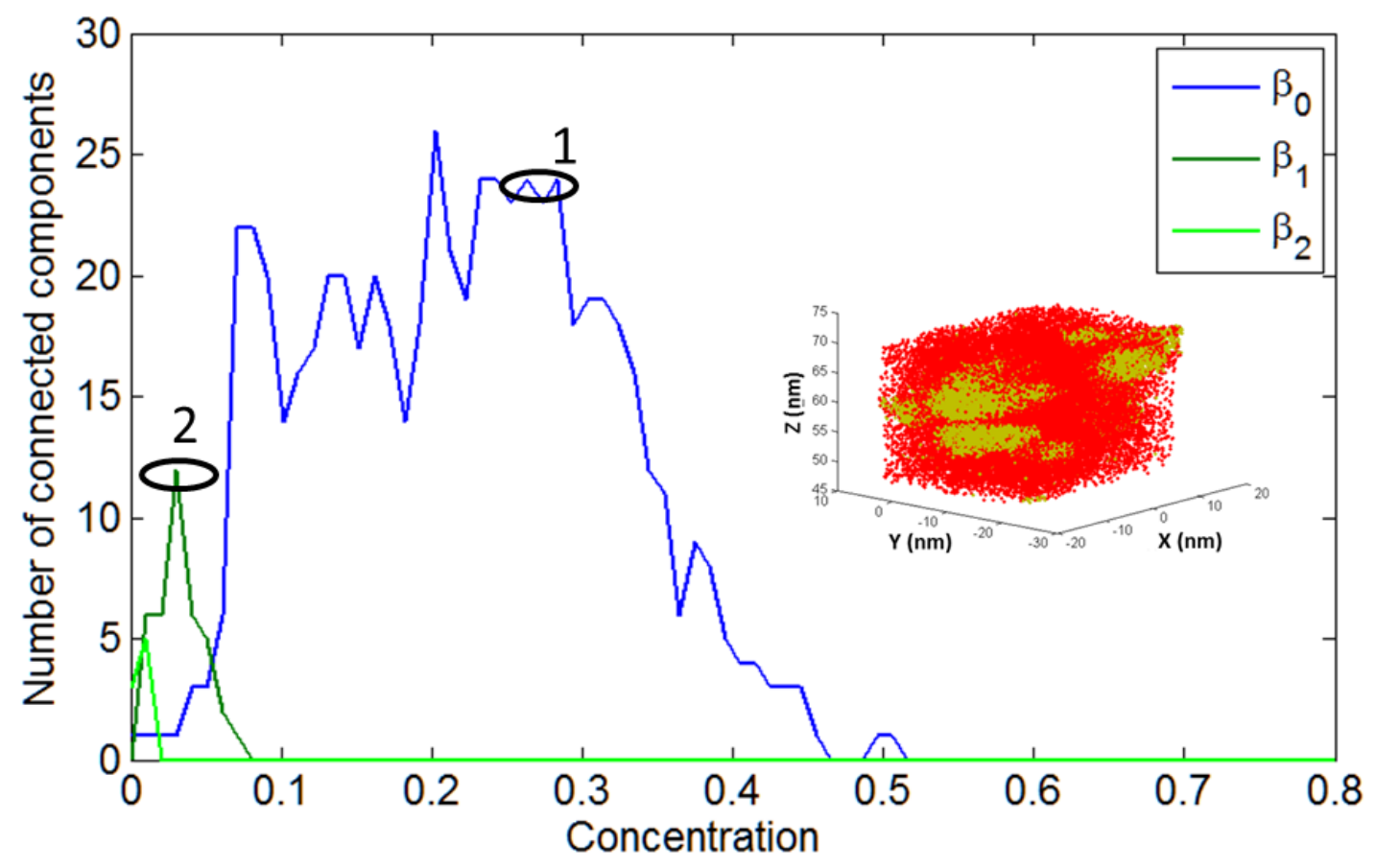

Figure 5: Filtration analysis of the Sc component in $\mathrm{Al}-\mathrm{Mg}-\mathrm{Sc}$ with respect to concentration threshold. (inset) The original sample specimen showing Sc (yellow) clusters embedded in $\mathrm{Mg}$ (red). The graph shows evolution of Betti numbers with changes in the concentration threshold for Sc. The set of $\left(\beta_{0}, \beta_{1}, \beta_{2}\right)$ captures the number of precipitates, tunnels and cavities formed by $\mathrm{Sc}$ for different concentration thresholds. Region 1 corresponds to persistence of $\beta_{0}$ at Sc concentration $\sim 0.3$ while region 2 in the graph points the concentration value $(\sim 0.03)$ for which $\beta_{1}$ shows a spike indicating the presence of hollow components. The corresponding voxel images for these two regions is shown in Fig. 6 and explained further in the text.

Consider $\beta_{0}$, the measure of simply connected components: at the higher end of concentration threshold, beyond 0.5 , a very small number of these are observed. This is because very few voxels have Sc concentration value equal or greater than this threshold. As concentration threshold is decreased, more voxels qualify to be included in the group leading to an increase in $\beta_{0}$. The value of $\beta_{0}$ remains constant for a certain range, labelled as region ' 1 ' in Fig. 5, indicating that these are real features due to their persistence. A scatter plot [Fig. 6b] of the voxels at $\delta=0.3$ shows that it indeed captures real clusters of Sc. The region 1 extends over a large concentration range because the Sc clusters comprise voxels with these various concentrations. With further decrease of concentration threshold, a decrease in $\beta_{0}$ is observed. This is because, below a certain value of Sc concentration, $\delta$, every voxel in the entire sample qualifies for consideration since it has some minimal content of Sc and the inclusion of all voxels results in one single connected component. We also observe a peak in the value of $\beta_{1}$ at a low concentration of $\delta=0.03$, labelled as region ' 2 ' in Fig. 5. When we extract the voxels corresponding to this Sc concentration value 
and plot [Fig. 6c] the isoconcentration surfaces relating these voxels, we find that they form partial cavities. These voxels with very low Sc concentration sit on the edge of the Sc clusters, and thereby, enclose Sc clusters within themselves. Region 1 illustrates spatial variance in the density distribution of Sc and the persistence of clusters at this broad range of concentrations indicate that these are real clusters and not statistical noise. Region 2, on the other hand, points to the precise boundary of the Sc clusters, thus, demarcating where the clusters lie in the sample. The computation of multiple Betti numbers allows additional degrees of freedom to independently confirm features with higher precision.

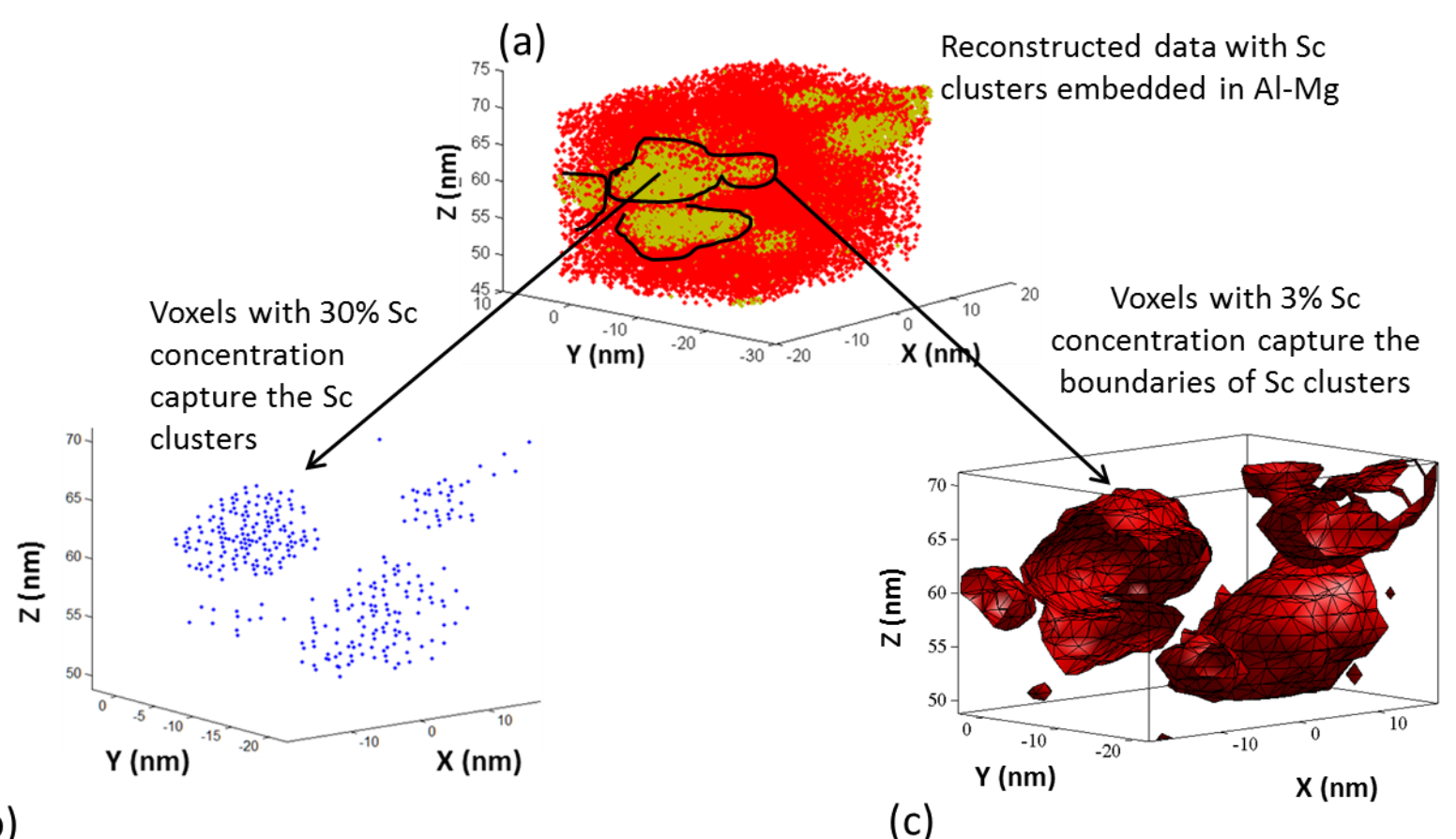

(b)

(c)

Figure 6: Recovering visual information based on the homology computation in Fig. 5 (a) The original sample specimen showing Sc (yellow) clusters embedded in Mg (red). (b) Voxels with Sc concentration 0.3, denoted by region 1 on Fig. 5, are shown to form Sc clusters while (c) voxels with Sc concentration 0.03 , denoted by region 2 in Fig. 5, form partial cavities/ tunnels representing the boundaries of the Sc clusters.

A similar trend is observed with $\mathrm{Mg}$ [Fig. 7a] where for low concentration we see $\mathrm{Mg}$ isosurface components distributed throughout the sample [Fig. 7b], whereas for high concentration there are few voxels. With Al, on the other hand, a trend reversal compared to Sc is observed [Fig. 7c]. This is because the Sc clusters are marked by a depletion of $\mathrm{Al}$ and conversely, the Sc concentration falls wherever there is enrichment of $\mathrm{Al}$. For a small value of $\mathrm{Al}$ concentration $\delta=(0-0.2)$ there are not many voxels that 
qualify and the $\beta_{0} \sim 0$. However, as the $\delta$ is increased the number of voxels with this concentration of Al increases and the number of connected components is almost uniform with some fluctuation. Beyond a certain value all the voxels get connected resulting in a single connected component. Interestingly for a high value of $\mathrm{Al}$, at $\delta=0.9$, we again find a spike in $\beta_{1}$. The isosurface corresponding to this value is shown in [Fig. 7d] as a blue mesh. This appears to correspond to the voxels that together enclose the Sc clusters, i.e., there appears to be some form of $\mathrm{Al}$ enrichment at the boundary of the Sc clusters. For clarity the voxels with high Sc concentration are shown embedded inside the Al mesh.

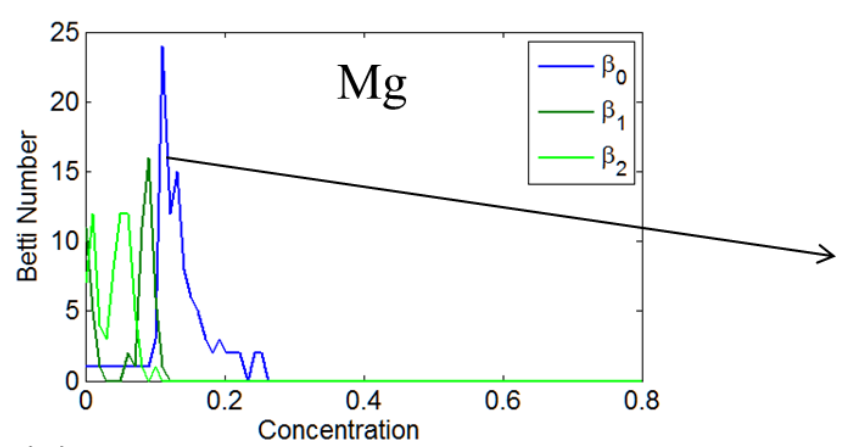

(a)

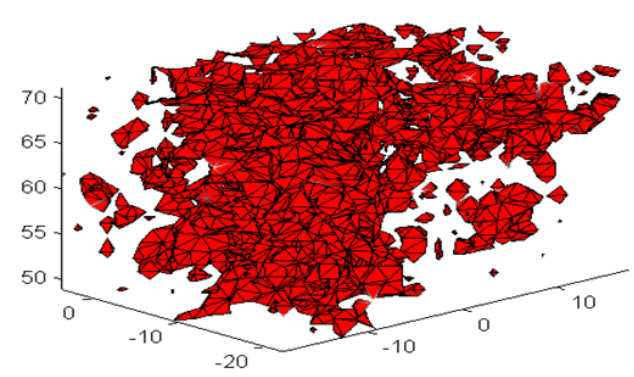

(b)

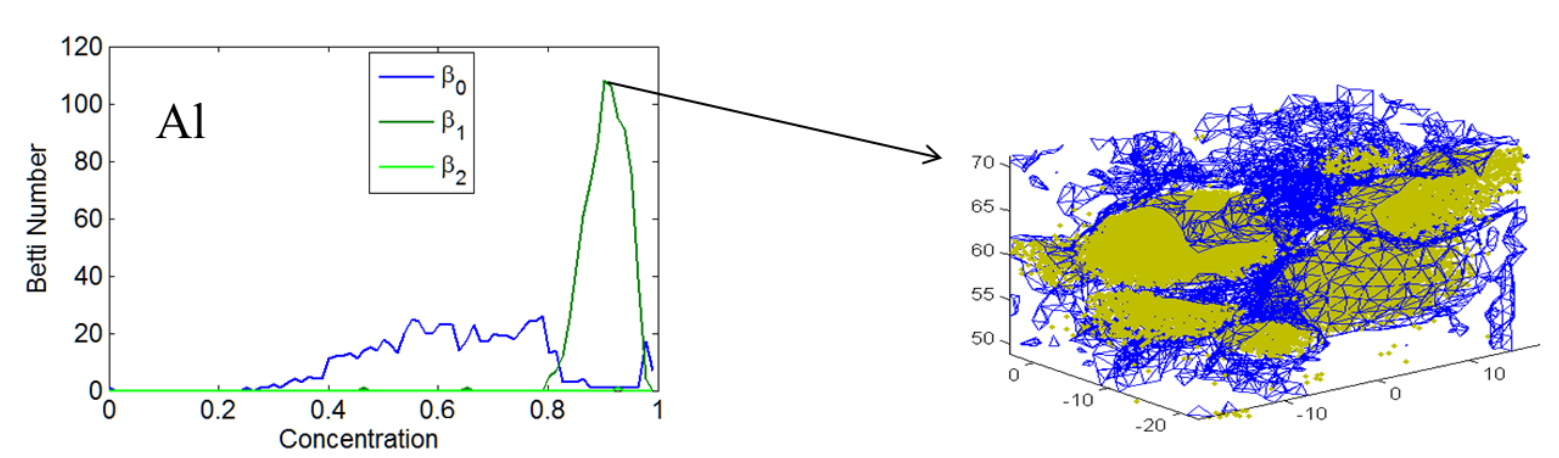

(c)

(d)

Figure 7: Filtration of the $\mathrm{Mg}$ and $\mathrm{Al}$ component in the $\mathrm{Al}-\mathrm{Mg}-\mathrm{Sc}$ structure with respect to concentration threshold. (a) $\mathrm{Mg}$ distribution in the sample follows the same trend as Sc. The voxels with maximum $\mathrm{Mg}$ concentration in the sample contain $\sim 25 \% \mathrm{Mg}$ beyond which $\beta_{0}=0$. (b) At lower concentration the $\mathrm{Mg}$ forms nanocluster envelopes scattered throughout. (c) $\mathrm{Al}$ on the other hand follows the opposite trend as $\mathrm{Sc}$ showing that (d) these two chemical components deplete each other in the alloy.

Comparing Figs. 6 and 7 we can clearly see that for Al concentration of 0.9 or Sc concentration of 0.03 , we are most likely capturing the same set of voxels, which lie at the boundary of the Sc clusters. One may 
also note that $\beta_{1}$ and $\beta_{2}$ often follow the same trend as $\beta_{0}$. This happens due to the fact that there is an optimal concentration level at and below which a chemical constituent arranges itself within the material, often determined by stoichiometry. When the concentration threshold reaches this point there is a high number of connected voxels or nanoclusters. Away from this concentration value, the voxels that qualify usually lie at the boundaries of these clusters and the interconnection of these form tunnels and cavities enclosing the high concentration regions within themselves.

Thus, by tracking the changes in Betti numbers with concentration threshold for individual chemical components of the material system, one can arrive at a precise understanding of how these components interact and arrange themselves. Instead of mining the entire data relying on visual acuity to probe for features, the data driven approach using Betti numbers provides a compact low dimensional representation of the sample's morphological information. The precise value of concentration threshold for extracting features from APT data can be determined by training oneself to read the Betti numbers graph and then examining specific features of interest.

By employing this approach we have uncovered new chemical and kinetic information on the precipitates and the corresponding interfaces that is missed when defining precipitates using predefined isoconcentration surfaces, which assume some precipitate shape and concentration threshold. First, the subtle $\mathrm{Al}$ segregation to the interface with $\mathrm{Sc}$ we have seen has not been previously discussed, even though this alloy has been studied previously [23, 24]. Further, by identifying the difference in precipitates from convex precipitates, the kinetic pathways leading to the formation of these precipitates can be further characterized. Similarly, precipitate volume can be more accurately measured now by a measure of the precise concentration value of the boundary, which can then be correlated with material behavior. Depending on the material system and how elements segregate there could be a clear demarcation allowing us to zero in on a specific concentration threshold or in some cases it may be diffuse and the appropriate choice may again require heuristic knowledge or user experience. Nevertheless this approach provides an additional layer of transparency because one can computationally keep track of all connected structures whether they are readily visible or not, thus, providing an objective estimate of the appropriate concentration threshold. The use of landmark points becomes necessary when evaluating the persistence of topological structures within the multi-million APT data on a single processor. To apply this technique on the entire dataset required parallelization strategies of this technique. 


\section{Conclusions}

This work has demonstrated the advantage of alternate data driven approaches for objectively uncovering different features, such as precise demarcation of Sc clusters and Al segregation at the cluster boundaries in $\mathrm{Al}-\mathrm{Mg}$-Sc alloys that can be visually obscured within the high volume of data concomitant with subnanometer resolution techniques such as APT. The use of simplicial homology provides a higher degree of transparency by substituting visual identification through a mathematically defined topology of the sample and computationally determining the presence of precipitates, tunnels and cavities in the sample. The application of persistence accounts for statistical uncertainty in the topological approach and minimizes the occurrence of artifacts.

\section{Acknowledgment}

We acknowledge support from the Air Force Office of Scientific Research (AFOSR) under grant numbers: FA9550-11-1-0158 and FA9550-12-1-0456; and support from NSF under grant numbers: PHY CDI-09-41576 and ARI Program CMMI-09-389018. KR also acknowledges support from the Wilkinson Professorship of Interdisciplinary Engineering.

\section{References}

1. Gault, B., et al., Atom probe microscopy. Vol. 160. 2012: Springer.

2. $\quad$ Miller, M.K., et al., Atom probe field ion microscopy. 1996.

3. Kelly, T.F. and M.K. Miller, Atom probe tomography. Review of Scientific Instruments, 2007. 78(3): p. 031101.

4. Seidman, D.N. and K. Stiller, An atom-probe tomography primer. MRS Bull, 2009. 34(10): p. 717-724.

5. Jiang, L., W. Chun-wu, and T.T. Tsong, Measurement of the atomic site specific binding energy of surface atoms of metals and alloys. Surface Science, 1991. 246(1-3): p. 157-162.

6. Hellman, O., et al., Application software for data analysis for three-dimensional atom probe microscopy. Materials Science and Engineering: A, 2002. 327(1): p. 29-33.

7. Stephenson, L.T., et al., New techniques for the analysis of fine-scaled clustering phenomena within atom probe tomography (APT) data. Microscopy and Microanalysis, 2007. 13(06): p. 448463. 
8. Shariq, A., et al., Exploring the next neighbourhood relationship in amorphous alloys utilizing atom probe tomography. Ultramicroscopy, 2007. 107(9): p. 773-780.

9. Geuser, F.D. and W. Lefebvre, Determination of matrix composition based on solute-solute nearest-neighbor distances in atom probe tomography. Microscopy research and technique, 2011. 74(3): p. 257-263.

10. Bryden, A., et al., Interactive visualization of APT data at full fidelity. Ultramicroscopy, 2013. 132(0): p. 129-135.

11. Broderick, S.R., et al., Data mining for isotope discrimination in atom probe tomography. Ultramicroscopy, 2013. 132: p. 121-128.

12. Samudrala, S., et al., A graph-theoretic approach for characterization of precipitates from atom probe tomography data. Computational Materials Science, 2013. 77: p. 335-342.

13. Hatcher, A., Algebraic topology. 2002: 清华大学出版社有限公司.

14. Zomorodian, A.J., Topology for computing. 2005: Cambridge University Press.

15. Eilenberg, S., Singular homology theory. Annals of Mathematics, 1944: p. 407-447.

16. Carlsson, G., Topology and data. Bulletin of the American Mathematical Society, 2009. 46(2): p. 255-308.

17. Rote, G. and G. Vegter, Computational Topology: An Introduction, in Effective Computational Geometry for Curves and Surfaces, J.-D. Boissonnat and M. Teillaud, Editors. 2006, Springer Berlin Heidelberg. p. 277-312.

18. Edelsbrunner, H., D. Letscher, and A. Zomorodian, Topological persistence and simplification. Discrete and Computational Geometry, 2002. 28(4): p. 511-533.

19. De Silva, V. and G. Carlsson. Topological estimation using witness complexes. in Proceedings of the First Eurographics conference on Point-Based Graphics. 2004. Eurographics Association.

20. Hetherington, M. and M. Miller, On the Statistical Analysis of Atom Probe Data. Le Journal de Physique Colloques, 1987. 48(C6): p. C6-559-C6-564.

21. Torres, K., et al., The influence of voxel size on atom probe tomography data. Ultramicroscopy, 2011. 111(6): p. 464-468.

22. Tausz, A.a.V.-J., Mikael and Adams, Henry, JavaPlex: A research software package for persistent (co)homology. 2011.

23. Gröbner, J., et al., Experimental investigations and thermodynamic calculation in the Al-Mg-Sc system. Zeitschrift für Metallkunde, 1999. 90(11): p. 872-880.

24. Marquis, E.A., et al., Composition evolution of nanoscale Al $<s u b>3</ s u b>$ Sc precipitates in an Al-Mg-Sc alloy: Experiments and computations. Acta materialia, 2006. 54(1): p. 119-130. 